\title{
Modelling of Vortex-Induced Aviation Turbulence
}

\author{
Nils T. Basse
}

Received: date / Accepted: date

\begin{abstract}
Aviation turbulence is modelled as an interaction between an aircraft and a vortex tube. The vortex tube can have an arbitrary orientation/offset with respect to the aircraft. We compare modelling the aircraft (i) as a point and (ii) having a finite area (wing and fuselage). We consider both vertical and horizontal acceleration experienced by the aircraft. The baseline vortex tube has an area which is of the order of the aircraft area.
\end{abstract}

Keywords Aviation turbulence · Vortex tube · Finite aircraft area · Vertical and horizontal acceleration

\section{Introduction}

Aviation turbulence (Sharman and Lane 2016) has an impact on flight comfort, safety and cost. A better understanding - and modelling - of the physical phenomena is needed to reduce the undesired effects of aviation turbulence. In this paper, we will assume that (a part of) aviation turbulence can be described as an interaction between an aircraft (AC) and a vortex tube (VT) (Lamb 1932; Saffman 1992). Our starting point is (Lunnon 2016).

Illustrations of how VTs can form are shown in e.g. Fig. 1 in (Wingrove and Bach 1994). The underlying instabilities are described in (Lin 2007). One candidate instability, the Kelvin-Helmholtz instability, is described in more detail in (Batchelor 1967) as "The instability of a sheet vortex".

Horizontal vortex tubes (HVTs) have been identified to occur during aviation turbulence (Clark et al. 2000). A physical mechanism for the formation of HVTs has been provided in (Roach 1970; Kaplan et al. 2004).

The main purpose of our paper is to extend the modelling efforts in Parks et al. 1985; Mehta 1987): In those papers, HVTs were modelled and the aircraft

Elsas väg 23, 42338 Torslanda, Sweden

Tel.: +46-705200362

E-mail: nils.basse@npb.dk 


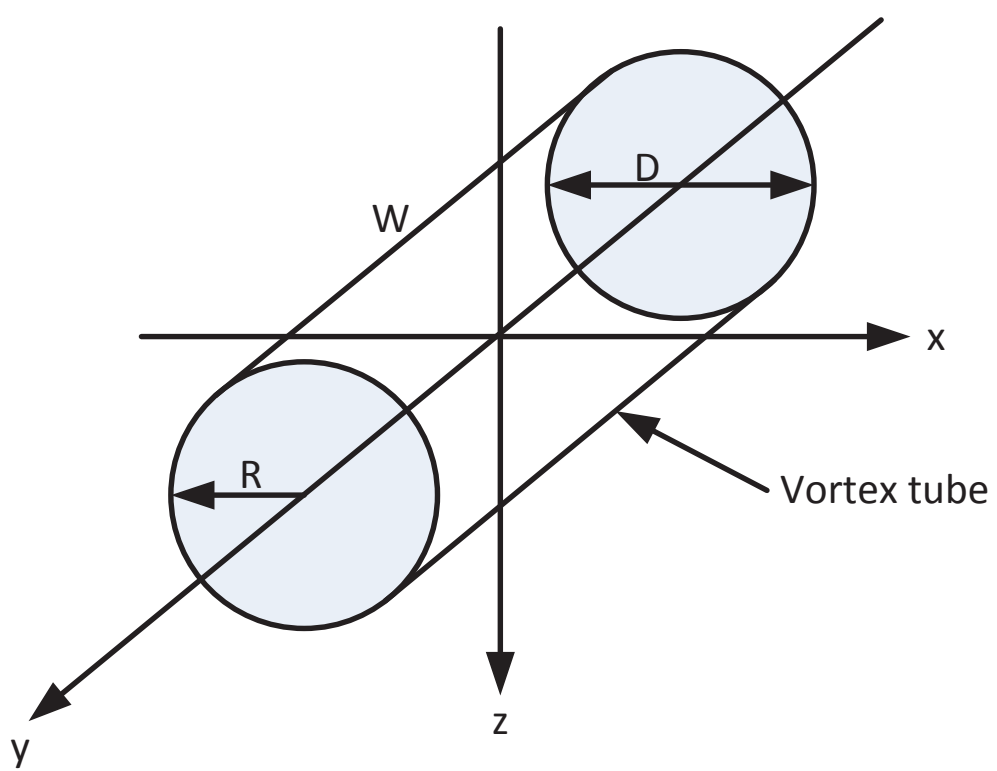

Fig. 1 Baseline vortex tube geometry and dimensions.

was assumed to be a point. Our extension includes VTs of arbitrary orientation/offset and a finite area of the aircraft (wing/fuselage). Further, we consider both vertical (normal) and horizontal (transverse) acceleration of the aircraft.

Traditionally the normal component of acceleration is regarded as the most important from the perspective of the safety of the aircraft - large normal accelerations can result in aircraft damage. From the perspective of passenger comfort, horizontal accelerations can cause injuries; this is reflected in the formulation of the Dose of Discomfort measure (Jacobson et al. 1978) in which all three components of acceleration are given equal weight.

\section{Modelling}

\subsection{Vortex Tube Properties}

The vortex is modelled as a circular $2 \mathrm{D}$ vortex extending into the third dimension. The baseline vortex is shown in Fig. 1 The vortex radius is $R$, the vortex diameter is $2 R=D$ and the vortex width is $W$. We note that a finite vortex width is not supported by theory, which states that vortex tubes either extend to infinity or end on solid boundaries (Saffman 1992). The vortex axis is along the $y$-direction.

The aircraft travels in the $x$-direction. The clockwise direction of the vortex velocity in the $x z$-plane leads to vorticity in the negative $y$-direction, see Fig. 2 


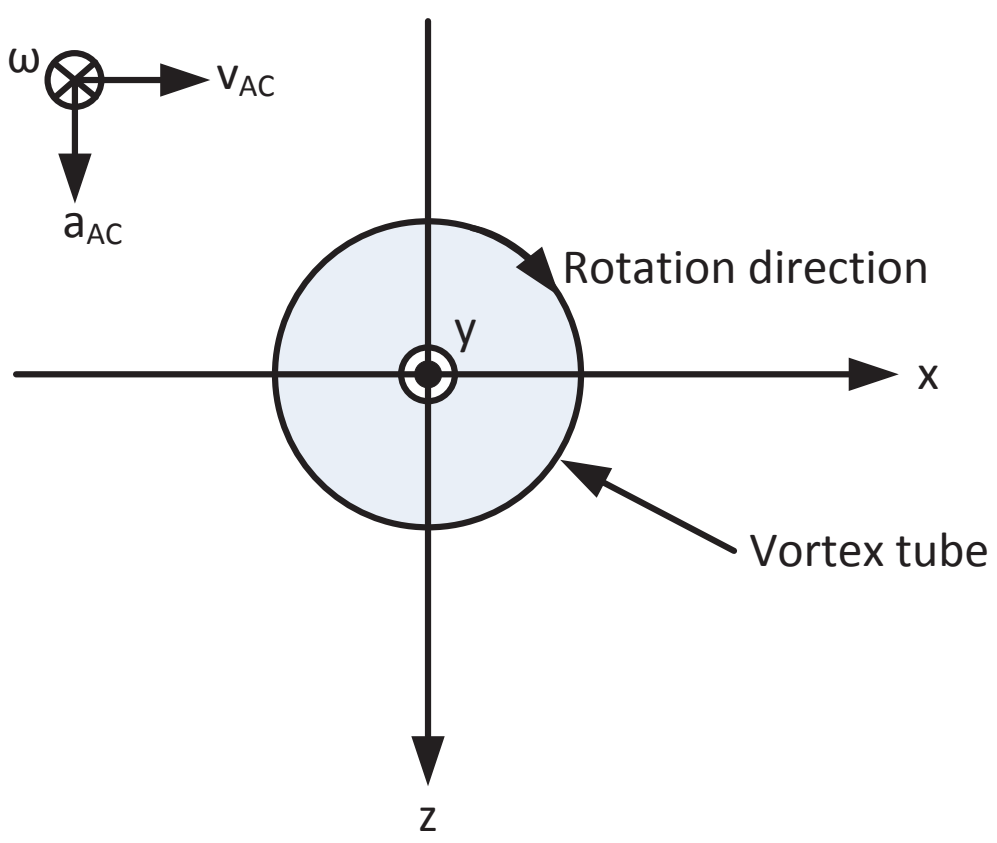

Fig. 2 Vortex tube velocity direction and resulting aircraft acceleration.

With respect to the aircraft, the $x$-direction is longitudinal, the $y$-direction is transverse and the $z$-direction is vertical. Since we will see that the acceleration is zero in the longitudinal direction, we will use the term horizontal for the transverse direction $(y)$.

We assume that the vortex rotates like a solid body with a constant angular velocity $\Omega$ :

$$
\boldsymbol{\Omega}=(0,-\Omega, 0)
$$

The vortex radius is $\boldsymbol{r}=(x, 0, z)$. The resulting vortex velocity is:

$$
\boldsymbol{u}=\boldsymbol{\Omega} \times \boldsymbol{r}=(-\Omega z, 0, \Omega x)
$$

The vorticity (vortex strength) is:

$$
\boldsymbol{\omega}=\nabla \times \boldsymbol{u}=(0,-2 \Omega, 0)=2 \boldsymbol{\Omega}
$$

For the general case we define the total vorticity as:

$$
\omega_{\mathrm{tot}} \equiv \sqrt{\omega_{x}^{2}+\omega_{y}^{2}+\omega_{x}^{2}}
$$

For the specific case, we have $\boldsymbol{\omega}=\left(0, \omega_{y}, 0\right)=(0,-2 \Omega, 0)$ and $\omega_{\text {tot }}=$ $\left|\omega_{y}\right|=2 \Omega$. 
The vortex speed is:

$$
u=r \Omega,
$$

where $r$ is the radius inside the vortex (from 0 to $R$ ).

The vortex circulation for radius $r$ is (Gerz et al. 2002):

$$
\Gamma(r)=\omega_{\text {tot }} r^{2} \pi=2 \Omega r^{2} \pi,
$$

so the total circulation of the vortex is:

$$
\Gamma_{\text {tot }}=\Gamma(R)=2 \Omega R^{2} \pi
$$

The vortex lifetime can be approximated by the eddy-turnover time (Pope 2000): Using $D$ as the length scale and $u(r=R)$ as the velocity, we get:

$$
\tau_{\text {vortex }}=D / u(r=R)=2 / \Omega
$$

\subsection{Aircraft Acceleration}

The main quantity we study is the aircraft acceleration due to a vortex tube as defined by Lunnon (Lunnon 2016):

$$
\mathbf{a}_{\mathrm{AC}}=\frac{1}{2} \boldsymbol{\omega} \times \mathbf{v}_{\mathrm{AC}}
$$

where $\mathbf{v}_{\mathrm{AC}}$ is the aircraft velocity, see Fig. 2.

Eq. (9) can be derived from the fluid (Lamb 1932; Batchelor 1967) (i.e. hydrodynamic (Saffman 1992)) impulse of the aircraft:

$$
\mathbf{I}_{\mathrm{AC}}=-\frac{1}{2} \rho_{\mathrm{AC}} \int \mathbf{r}_{\mathrm{AC}} \times \boldsymbol{\omega} \mathrm{d} V_{\mathrm{AC}},
$$

where $\mathbf{r}_{\mathrm{AC}}$ is the aircraft position, $\rho_{\mathrm{AC}}=\frac{m_{\mathrm{AC}}}{V_{\mathrm{AC}}}$ is the aircraft density, $m_{\mathrm{AC}}$ is the aircraft mass and $V_{\mathrm{AC}}$ is the aircraft volume. Note that $m_{\mathrm{AC}}$ and $V_{\mathrm{AC}}$ are defined for the part of the aircraft which is enclosed by the vortex.

Here, we have modified the original expression in two ways:

- We have introduced a negative sign to account for the fact that we study the impulse of the aircraft instead of the impulse of the vortex

- We have replaced vortex by aircraft quantities where relevant

We assume that the vorticity of the vortex is independent of time. Thus, we can take the time derivative of the impulse to arrive at the force on the aircraft:

$$
\begin{aligned}
\mathbf{F}_{\mathrm{AC}}=\frac{\mathrm{d} \mathbf{I}_{\mathrm{AC}}}{\mathrm{d} t}=m_{\mathrm{AC}} \mathbf{a}_{\mathrm{AC}} & =-\frac{1}{2} \frac{m_{\mathrm{AC}}}{V_{\mathrm{AC}}} \int \mathbf{v}_{\mathrm{AC}} \times \boldsymbol{\omega} \mathrm{d} V_{\mathrm{AC}} \\
\mathbf{a}_{\mathrm{AC}} & =\frac{1}{V_{\mathrm{AC}}} \int \frac{1}{2} \boldsymbol{\omega} \times \mathbf{v}_{\mathrm{AC}} \mathrm{d} V_{\mathrm{AC}}
\end{aligned}
$$


Since the aircraft velocity is constant, we can remove the integral; this concludes our derivation of Eq. (9).

We assume that the vortex is stationary and that the aircraft is moving. If the vortex is moving, the aircraft velocity is interpreted as the velocity difference between the aircraft and the vortex.

We assume that the acceleration will not move the aircraft, i.e. the aircraft trajectory is fixed.

Using Eq. (9) and the fact that the aircraft is travelling in the $x$-direction we find:

$$
\mathbf{a}_{\mathrm{AC}}=\frac{1}{2}(0,-2 \Omega, 0) \times\left(v_{\mathrm{AC}}, 0,0\right)=\left(0,0, \Omega v_{\mathrm{AC}}\right)
$$

Thus, the aircraft experiences an acceleration in the positive $z$-direction, see Fig. 2 .

\subsection{Example}

We use a typical cruising speed $v_{\mathrm{AC}}=800 \mathrm{~km} / \mathrm{h}=800 / 3.6 \mathrm{~m} / \mathrm{s}=222.2 \mathrm{~m} / \mathrm{s}$.

From (Sharman 2016), we set the aircraft acceleration of a significant event to $a_{\mathrm{AC}}=0.5 \mathrm{~g}=4.9 \mathrm{~m} / \mathrm{s}^{2}$.

Combining the above, the angular velocity of the vortex $\Omega=a_{\mathrm{AC}} / v_{\mathrm{AC}}=$ $2.2 \times 10^{-2} \mathrm{~s}^{-1}$. The resulting vorticity $\omega_{y}=-2 \Omega=-4.4 \times 10^{-2} \mathrm{~s}^{-1}$. 2.4 .3 .

We choose a vortex radius $R=10.7 \mathrm{~m}$ based on the discussion in Section

This leads to a maximum speed on the vortex surface $u(r=R)=R \Omega=$ $0.24 \mathrm{~m} / \mathrm{s}$.

The total circulation of the vortex is $\Gamma_{\text {tot }}=15.9 \mathrm{~m}^{2} / \mathrm{s}$.

The eddy-turnover time $D / u(r=R)=2 / \Omega=91 \mathrm{~s}$. This can be compared to the duration when the aircraft is inside the vortex $D / v_{\mathrm{AC}}=0.1 \mathrm{~s}$. For this case, the vortex persists much longer than the aircraft takes to pass through it, so we do not have to consider vortex decay. However, vortex lifetime will become increasingly important for larger $\Omega$ and/or slower aircraft.

\subsection{Aircraft Geometry}

\subsubsection{Point}

The simplest aircraft model is to consider it as a point. The implicit assumption is that the vortex dimensions are much larger than the aircraft dimensions. We will discuss the errors using this approach in more detail below. 


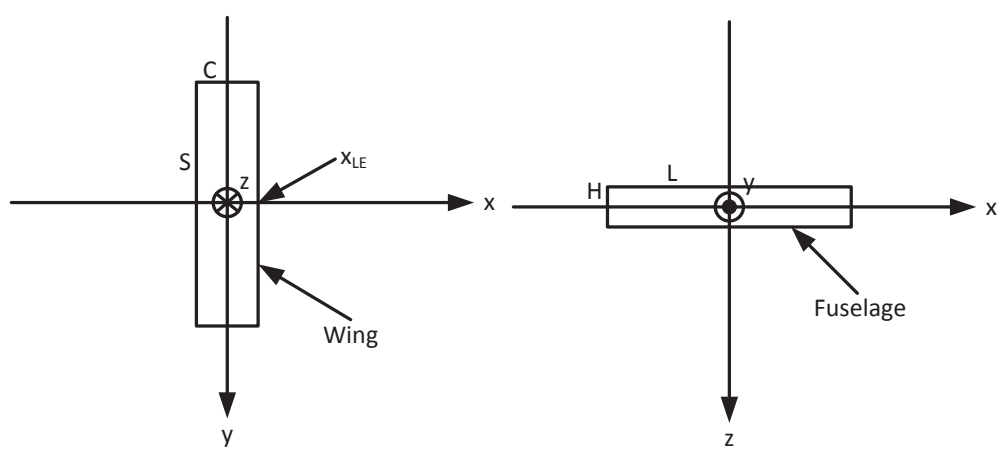

Fig. 3 Aircraft geometry: Wing (left) and fuselage (right).

\subsubsection{Area}

When we model the aircraft with a finite area, we use dimensions approximating those of an Airbus A330-200 (Airbus 2019), i.e. a medium-sized aircraft.

The wing span $S$ is fixed at $60 \mathrm{~m}$ and the wing chord $C$ is set to $6 \mathrm{~m}$, see Fig. 3. The position of the leading edge (LE) of the wing is shown as $x_{\mathrm{LE}}$. The fuselage length $L$ is $60 \mathrm{~m}$ and the fuselage height $H$ is $6 \mathrm{~m}$.

The wing is assumed to be fixed exactly at the center of the fuselage. Both the wing and fuselage are assumed to be rectangles. The horizontal stabilizer and tail fin are not considered.

It would be more realistic to represent the fuselage as a cylinder rather than a rectangle - the cross section of the fuselage of an Airbus A330-200 is essentially circular. This could be achieved by adding a second horizontal rectangle, coaxial with the vertical rectangle which represents the fuselage, in the computations. However, the approach used is justified by being the simplest aircraft representation for which both normal and transverse accelerations can be derived.

\subsubsection{Point Versus Area}

To discuss the comparison between the vortex and aircraft areas, we define the vortex/aircraft area ratio $r_{A}$. For the fuselage, the vortex $x z$-plane area is $\pi R^{2}$ and the aircraft fuselage area is $L H$. For a fixed $r_{A}$, the vortex radius $R_{A}$ is given:

$$
\begin{aligned}
r_{A} & =\frac{\pi R_{A}^{2}}{L H} \\
R_{A} & =\sqrt{\frac{r_{A} L H}{\pi}}
\end{aligned}
$$

As for the fuselage, we also require that $r_{A}$ is equal to the ratio of the vortex $x y$-plane area $D W=2 R W$ and the aircraft wing area $S C$ : 


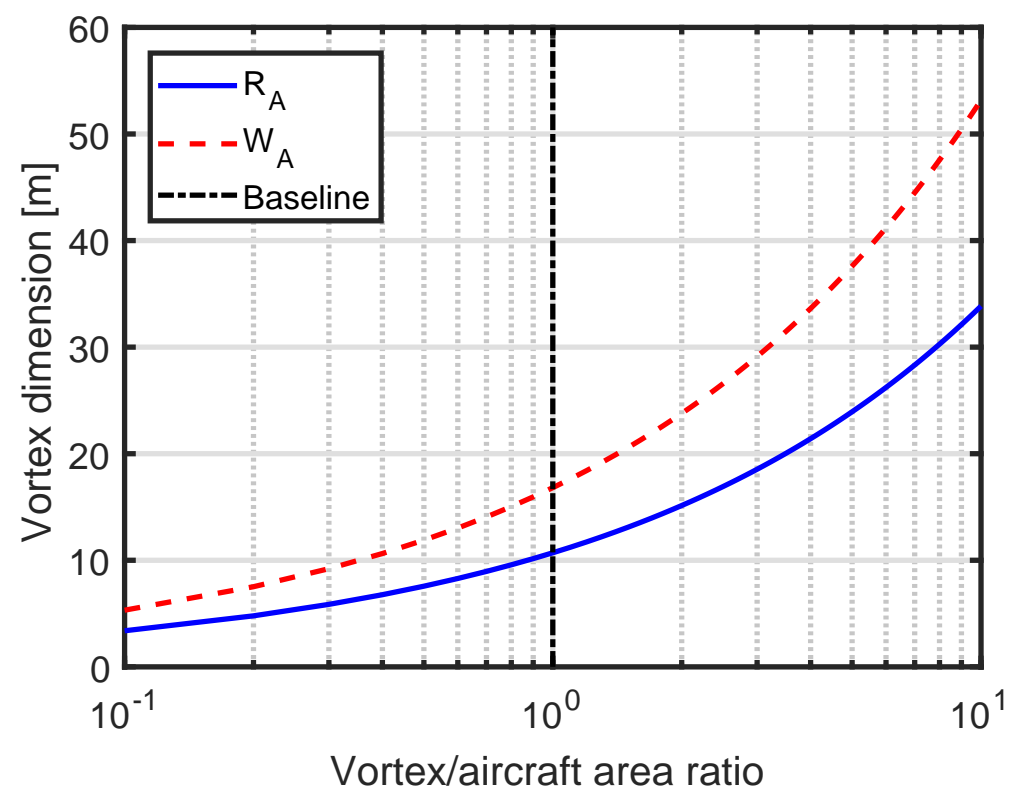

Fig. 4 Vortex dimensions as a function of vortex/aircraft area ratio.

$$
\begin{aligned}
r_{A} & =\frac{2 R_{A} W_{A}}{S C} \\
W_{A} & =\frac{r_{A} S C}{2 R_{A}}
\end{aligned}
$$

Combining Eqs. (15) and (17), we write the vortex width $W_{A}$ as:

$$
W_{A}=\frac{S C}{2} \sqrt{\frac{r_{A} \pi}{L H}}
$$

We can now use $r_{A}$ to determine the area ratio; this is illustrated in Fig. 4 for the aircraft dimensions provided above, where $R_{A}$ and $W_{A}$ are shown as a function of $r_{A}$. We will consider three cases below:

$-r_{A}=0.1(R=3.4 \mathrm{~m}, W=5.3 \mathrm{~m})$ : Vortex area is much smaller than aircraft area

$-r_{A}=1(R=10.7 \mathrm{~m}, W=16.8 \mathrm{~m})$ : Vortex area is the same as the aircraft area (our baseline case)

$-r_{A}=10(R=33.9 \mathrm{~m}, W=53.2 \mathrm{~m})$ : Vortex area is much larger than aircraft area

For the first two cases, the aircraft should be modelled with finite areas. For the last case, modelling the aircraft as a point is sufficient for most purposes. 

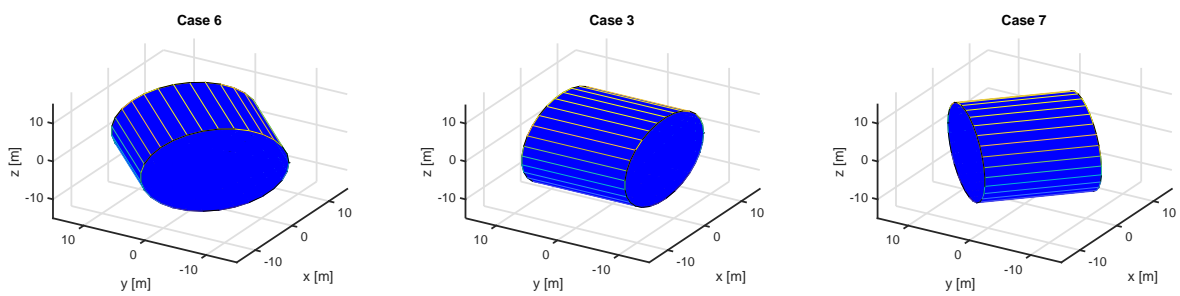

Fig. 5 Vortex tube orientation for left-right tilt: $\phi=\pi / 4$ (left), $\phi=\pi / 2$ (center), $\phi=3 \pi / 4$ (right).

\section{Results: Aircraft as a Point}

Here, we discuss analytical modelling of the aircraft as a point.

\subsection{Vortex Offset}

The vortex can be offset in the horizontal direction $y_{0}$. When $-W / 2<y_{0}<$ $W / 2$, the aircraft will be inside the vortex, otherwise it will be outside.

For a vertical offset $z_{0}$, the aircraft will travel a distance $2 \sqrt{R^{2}-z_{0}^{2}}$ inside the vortex. For example, if we want to reduce the distance from $2 R$ to $R$, we set $z_{0}=R \sqrt{\frac{3}{4}}$.

\subsection{Vortex Angle}

\subsubsection{Left-Right Tilt}

A left-right tilt of the vortex is rotation in the $x y$-plane. This is defined using the azimuthal angle $\phi$, where $\pi / 2$ is the baseline case. See Fig. 5 for an illustration of the corresponding vortex tube orientation.

The aircraft will travel a distance $2 R / \sin (\phi)$ inside the vortex.

The vorticity is given by:

$$
\begin{aligned}
\boldsymbol{\omega} & =\left(\omega_{x}, \omega_{y}, 0\right) \\
\omega_{x} & =-2 \Omega \cos \phi \\
\omega_{y} & =-2 \Omega \sin \phi
\end{aligned}
$$

The resulting aircraft acceleration is in the vertical direction:

$$
\mathbf{a}_{\mathrm{AC}}=\frac{1}{2}\left(\omega_{x}, \omega_{y}, 0\right) \times\left(v_{\mathrm{AC}}, 0,0\right)=\frac{1}{2}\left(0,0,-\omega_{y} v_{\mathrm{AC}}\right)
$$



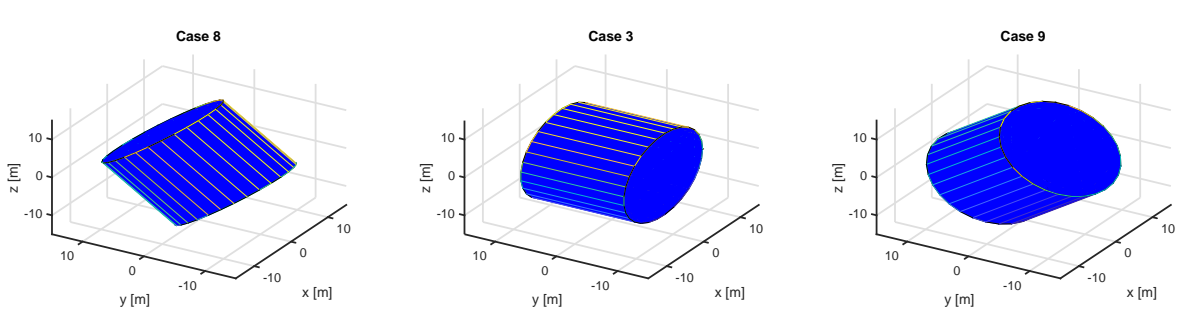

Fig. 6 Vortex tube orientation for up-down tilt: $\theta=\pi / 4$ (left), $\theta=\pi / 2$ (center), $\theta=3 \pi / 4$ (right).

\subsubsection{Up-Down Tilt}

An up-down tilt of the vortex is rotation in the $x z$-plane. This is defined using the polar angle $\theta$, where $\pi / 2$ is the baseline case. See Fig. 6 for an illustration of the corresponding vortex tube orientation.

The aircraft will travel a distance $2 R$ inside the vortex.

The vorticity is given by:

$$
\begin{aligned}
\boldsymbol{\omega} & =\left(0, \omega_{y}, \omega_{z}\right) \\
\omega_{y} & =-2 \Omega \sin \theta \\
\omega_{z} & =-2 \Omega \cos \theta
\end{aligned}
$$

The resulting aircraft acceleration is in both the horizontal and the vertical direction:

$$
\mathbf{a}_{\mathrm{AC}}=\frac{1}{2}\left(0, \omega_{y}, \omega_{z}\right) \times\left(v_{\mathrm{AC}}, 0,0\right)=\frac{1}{2}\left(0, \omega_{z} v_{\mathrm{AC}},-\omega_{y} v_{\mathrm{AC}}\right)
$$

\subsubsection{Combined Tilt}

For a combined tilt, both $\phi$ and $\theta$ are allowed to vary between 0 and $\pi$.

The vorticity is given by:

$$
\begin{aligned}
& \omega_{x}=-2 \Omega \sin \theta \cos \phi \\
& \omega_{y}=-2 \Omega \sin \theta \sin \phi \\
& \omega_{z}=-2 \Omega \cos \theta
\end{aligned}
$$

The resulting aircraft acceleration is in both the horizontal and the vertical direction:

$$
\mathbf{a}_{\mathrm{AC}}=\frac{1}{2}\left(\omega_{x}, \omega_{y}, \omega_{z}\right) \times\left(v_{\mathrm{AC}}, 0,0\right)=\frac{1}{2}\left(0, \omega_{z} v_{\mathrm{AC}},-\omega_{y} v_{\mathrm{AC}}\right)
$$

For values from our example above, the acceleration in the vertical and horizontal direction is shown as contour plots in Fig. 7. 

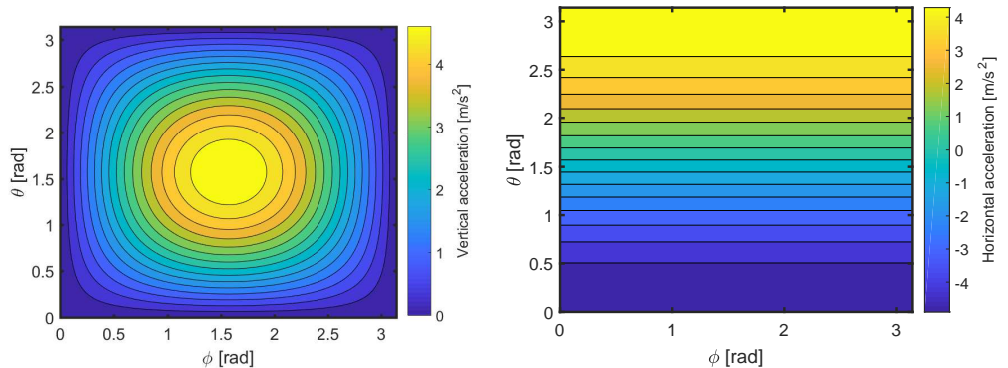

Fig. 7 Left: Vertical acceleration, right: Horizontal acceleration.

The sign of the vertical acceleration is always positive and is symmetric around the baseline orientation $(\phi, \theta)=(\pi / 2, \pi / 2)$ (which is the maximum acceleration).

The horizontal acceleration has a positive maximum for $\theta=\pi$ and a negative minimum for $\theta=0$. The horizontal acceleration is independent of $\phi$.

\section{Results: Aircraft as an Area}

\subsection{Numerical Procedure}

The numerical procedure when considering the aircraft to be an area consists of two steps. The first step is the generation of the vortex tube:

1. Create VT (split in 30 segments)

2. Apply offsets $y_{0}$ and $z_{0}$

3. Calculate convex hull of VT

4. Check that the volume of the convex hull is the same as the VT volume

5 . Define grids for the $x y$ - and $x z$-planes (resolution $0.5 \mathrm{~m}$ )

6. Calculate intersection between the VT and the two planes

7. Calculate the convex hull of the VT in the two planes

Once the VT is defined, the position of the aircraft is a function of time. We use 71 timesteps with a step length of $0.01 \mathrm{~s}$, i.e. a total duration of $0.7 \mathrm{~s}$. Using the aircraft speed from our example in Section 2.3 , this is equivalent to a distance of $156 \mathrm{~m}$.

The second step proceeds as follows:

1. Define $x_{\mathrm{LE}}$ for the given timestep

2. Define both the wing and the fuselage as four point polygons

3. Define the aircraft center point

4. Calculate the intersection between the VT in the $x y$-plane ( $x z$-plane) and the wing (fuselage), respectively

5. Check if center point is inside the VT

6. Calculate the ratio between the intersection area and the total wing (fuselage) area, respectively 

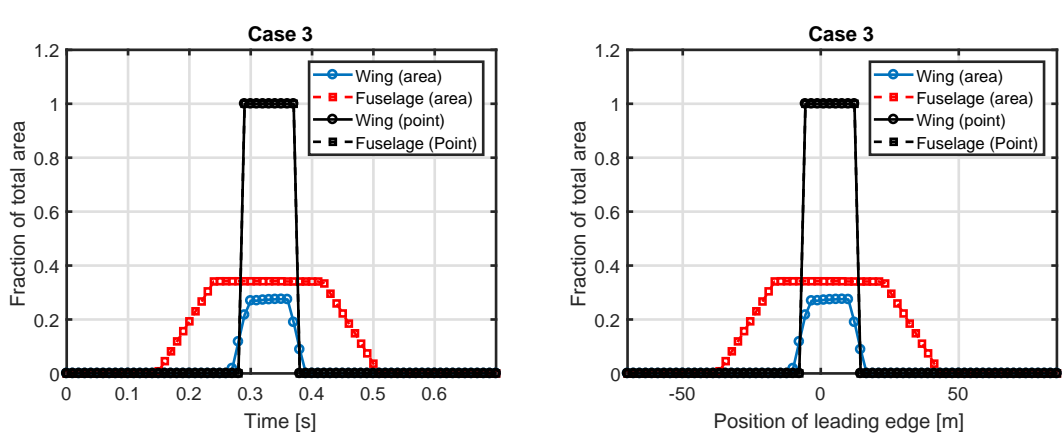

Fig. 8 Baseline vortex (case 3): Area fraction as function of time (left) and LE position (right). When considering the aircraft as a point, the wing and fuselage area fractions are identical.

Table 1 Table of cases. Case 3 is the baseline case.

\begin{tabular}{ccccccc}
\hline Case no & $\phi[\mathrm{rad}]$ & $\theta[\mathrm{rad}$ & $y_{0}[\mathrm{~m}]$ & $z_{0}[\mathrm{~m}]$ & $R[\mathrm{~m}]$ & $W[\mathrm{~m}]$ \\
\hline 1 & $\pi / 2$ & $\pi / 2$ & 0 & 0 & 33.9 & 53.2 \\
2 & $\pi / 2$ & $\pi / 2$ & 0 & 0 & 3.4 & 5.3 \\
3 & $\pi / 2$ & $\pi / 2$ & 0 & 0 & 10.7 & 16.8 \\
4 & $\pi / 2$ & $\pi / 2$ & 8.4 & 0 & 10.7 & 16.8 \\
5 & $\pi / 2$ & $\pi / 2$ & 0 & 9.3 & 10.7 & 16.8 \\
6 & $\pi / 4$ & $\pi / 2$ & 0 & 0 & 10.7 & 16.8 \\
7 & $3 \pi / 4$ & $\pi / 2$ & 0 & 0 & 10.7 & 16.8 \\
8 & $\pi / 2$ & $\pi / 4$ & 0 & 0 & 10.7 & 16.8 \\
9 & $\pi / 2$ & $3 \pi / 4$ & 0 & 0 & 10.7 & 16.8 \\
10 & $\pi / 4$ & $\pi / 4$ & 0 & 0 & 10.7 & 16.8 \\
11 & $\pi / 4$ & $3 \pi / 4$ & 0 & 0 & 10.7 & 16.8 \\
12 & $3 \pi / 4$ & $\pi / 4$ & 0 & 0 & 10.7 & 16.8 \\
13 & $3 \pi / 4$ & $3 \pi / 4$ & 0 & 0 & 10.7 & 16.8 \\
14 & 0 & $\pi / 2$ & 0 & 0 & 10.7 & 16.8 \\
15 & $\pi$ & $\pi / 2$ & 0 & 0 & 10.7 & 16.8 \\
16 & $\pi / 2$ & 0 & 0 & 0 & 10.7 & 16.8 \\
17 & $\pi / 2$ & $\pi$ & 0 & 0 & 10.7 & 16.8 \\
\hline
\end{tabular}

7. Multiply the maximum acceleration $(0.5 \mathrm{~g})$ by the area ratios

The area fractions are shown both as a function of time and leading edge position for our baseline case in Fig. 8, When considering the aircraft as a point, the area fraction is either zero (aircraft center point outside VT) or one (aircraft center point inside VT). This behaviour is different when the aircraft area is considered. Compared to the wing, the fuselage enters (exits) the VT first (last), respectively. It is also important to note that the area ratios are only about one-third of the point ratios, since the VT only intersects part of the aircraft.

All the cases we have modelled can be found in Table 1. 

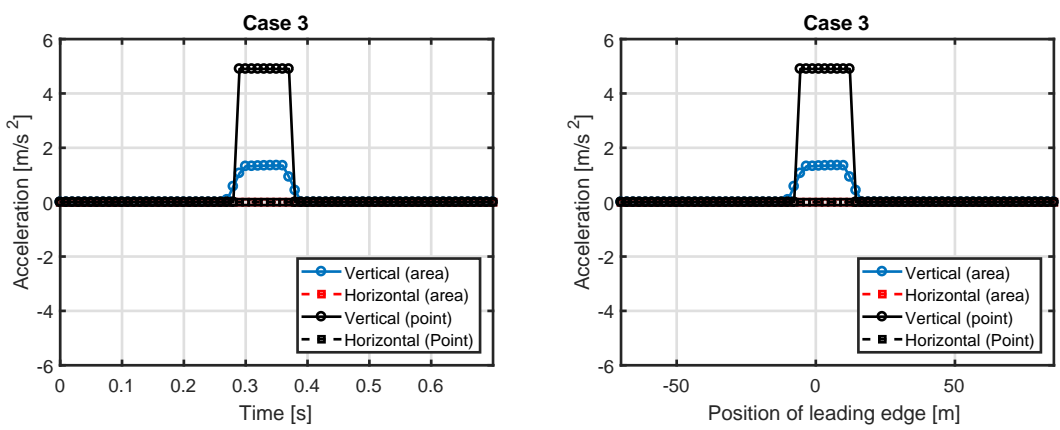

Fig. 9 Baseline vortex (case 3): Acceleration as function of time (left) and LE position (right). The horizontal acceleration is zero.

\subsection{Baseline}

Aircraft accelerations corresponding to the area ratios in Fig. 8 are shown in Fig. 9. First of all we note that there is no horizontal acceleration, consistent with our results in Section 3. The vertical area acceleration is about one-third of the acceleration if one considers the aircraft as a point, see our discussion above. Further, the increase/decrease of acceleration for the vertical acceleration as an area is not instantaneous, since the wing has a finite width, the chord $C$.

This result indicates that it is important to take the aircraft area into account.

For the following analysis, we only show acceleration as a function of time.

\subsection{Size}

Once we have established the baseline case, we can use the modelling procedure to gain insight into the impact of other VT geometries.

First we vary the VT size, see Fig. 10. These are cases 1, 2 and 3 in Table 1. The sizes are determined in Section 2.4.3

As the vortex size decreases, we note that the amplitude of the area acceleration decreases. This confirms that considering the aircraft as a point works well for a large VT, but not for a smaller VT.

\subsection{Offset}

We continue the modelling by investigating the effect of an offset VT, see Fig. 11. These are cases 3, 4 and 5. Moving the VT by $R$ in the horizontal direction means that the point acceleration is zero, i.e. the aircraft center point is exactly at the edge of the VT. A vertical VT offset by $R \sqrt{\frac{3}{4}}$ (see Section 3.1) means that the VT extent in the $x$-direction is reduced from $2 R$ to $R$. This decrease manifests itself as a shorter duration of the aircraft-VT encounter. 

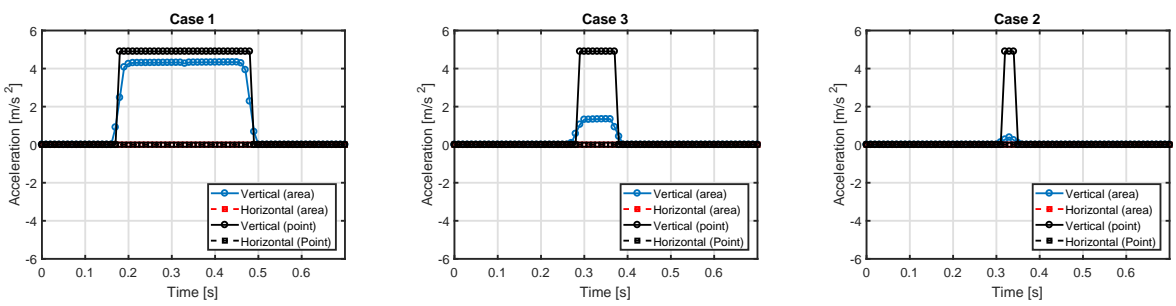

Fig. 10 Acceleration as function of time for vortex size variation: Large vortex (left), baseline vortex (center), small vortex (right). The horizontal acceleration is zero.
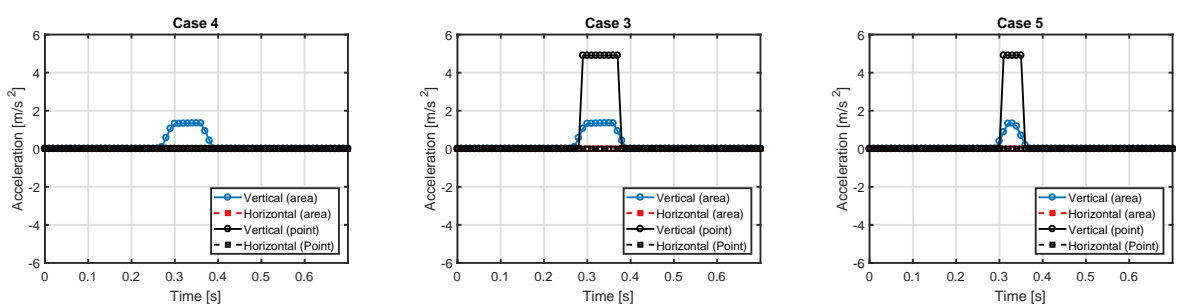

Fig. 11 Acceleration as function of time for vortex offset variation: Horizontal offset (left), no offset (center), vertical offset (right). The horizontal acceleration is zero.
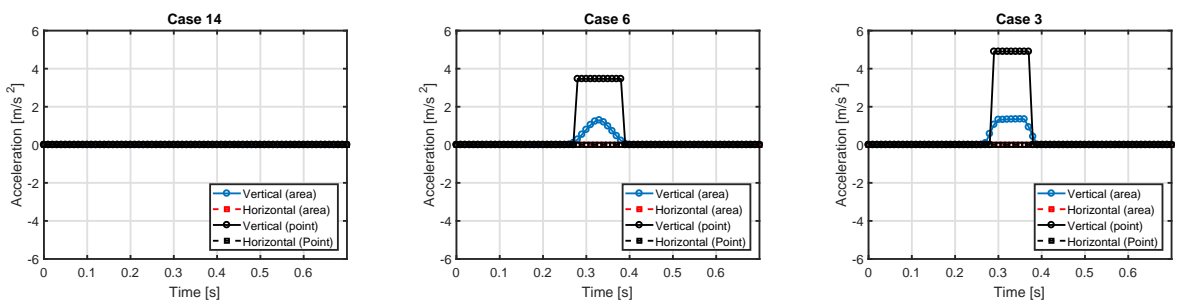

Fig. 12 Acceleration as function of time for vortex $\phi$ variation: $\phi=0$ or $\phi=\pi$ (left), $\phi=\pi / 4$ or $\phi=3 \pi / 4$ (center), $\phi=\pi / 2$ (right). The horizontal acceleration is zero.

4.5 Angle: $\theta=\pi / 2, \phi$ Varies

Here, we present results for the left-right tilt of the VT using the angle $\phi$, see Fig. 12. There is no up-down tilt, $\theta$ is kept fixed at $\pi / 2$. These are cases 3,6 and 14 .

If the VT is parallel to the aircraft, there is no acceleration. At an angle $\phi=\pi / 4$ (or $3 \pi / 4$ ), the area acceleration does not have a flat top and the duration of the aircraft-VT encounter is longer.

\subsection{Angle: $\phi=\pi / 2, \theta$ Varies}

For the up-down tilt, there is no left-right tilt, i.e. $\phi=\pi / 2$.

We first present the results for $\theta$ less than $\pi / 2$, see Fig. 13. These are cases 3,8 and 16 . An angle $\theta=0$ corresponds to a columnar vortex; here, only a 

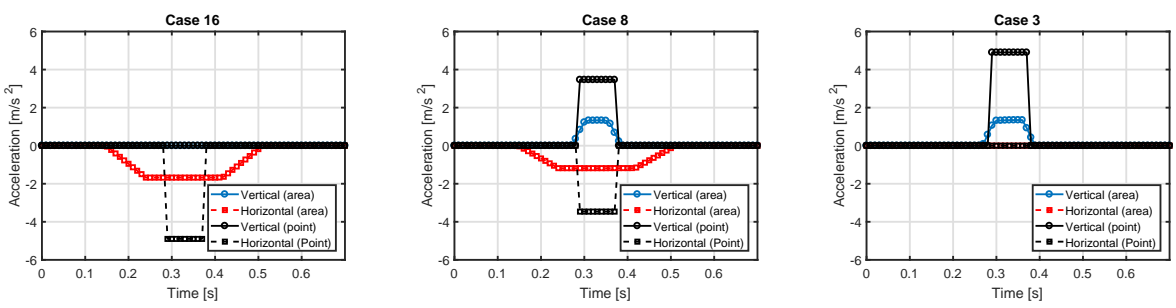

Fig. 13 Acceleration as function of time for vortex $\theta$ variation: $\theta=0$ (left, no vertical acceleration), $\theta=\pi / 4$ (center), $\theta=\pi / 2$ (right, no horizontal acceleration).
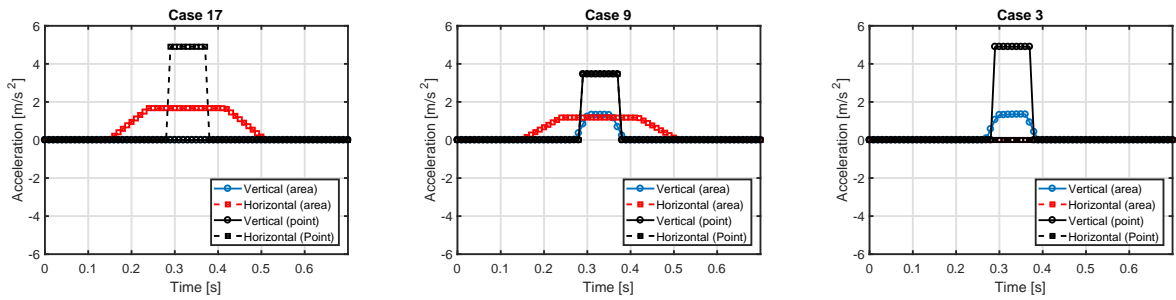

Fig. 14 Acceleration as function of time for vortex $\theta$ variation: $\theta=\pi$ (left, no vertical acceleration), $\theta=3 \pi / 4$ (center), $\theta=\pi / 2$ (right, no horizontal acceleration). For $\theta=3 \pi / 4$ (center), the vertical and horizontal acceleration is identical when considering the aircraft as a point.

horizontal acceleration takes place. The amplitude is lower and duration longer than when modelling the aircraft as a point. Both horizontal and vertical accelerations are present for $\theta=\pi / 4$.

We now proceed to present the results for $\theta$ larger than $\pi / 2$, see Fig. 14 These are cases 3,9 and 17 . The vertical acceleration has the same amplitude and direction as for the smaller angles. The horizontal acceleration has the same amplitude but the opposite sign.

\subsection{Angle: Both Angles Vary}

Removing the restricting of a fixed $\phi$ or $\theta$ we can consider other combinations, see Fig. 15. For these cases, the amplitude of the vertical and horizontal acceleration differs.

\section{Discussion and Future Work}

The model we have presented above is a starting point; it is relatively straightforward to extend our model.

One example is asymmetry effects: The left/right wing and forward/aft fuselage can be treated separately. As an illustration we show the offset cases (4 and 5) in Fig. 16. Because of the fuselage length, it seems likely that there 

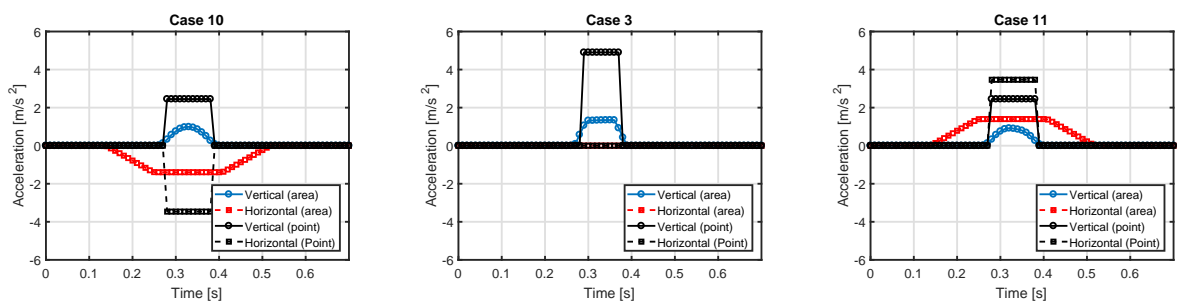

Fig. 15 Acceleration as function of time for vortex combined angle variation: $\phi=\pi / 4, \theta=$ $\pi / 4$ or $\phi=3 \pi / 4, \theta=\pi / 4$ (left), $\phi=\pi / 2, \theta=\pi / 2$ (center, no horizontal acceleration), $\phi=\pi / 4, \theta=3 \pi / 4$ or $\phi=3 \pi / 4, \theta=3 \pi / 4$ (right).
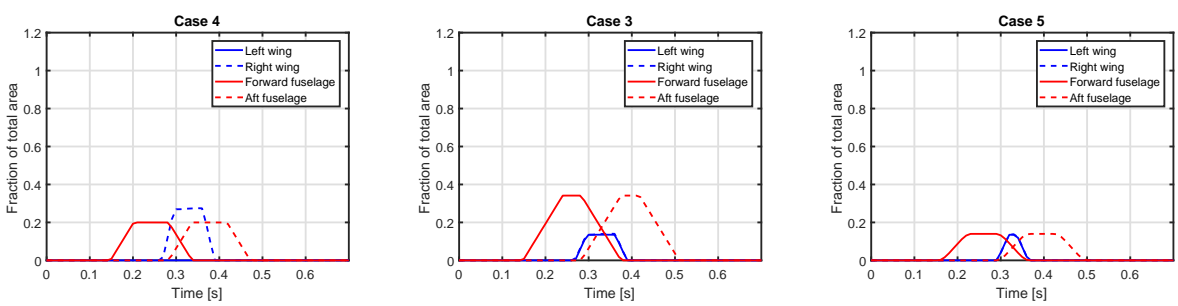

Fig. 16 Area fraction as function of time: Horizontal offset (left), no offset (center), vertical offset (right). For the left-hand plot, the area fraction of the left wing is zero; for the center and right-hand plots, the left and right wing ratios are identical.

will be significant stress between the forward and aft part compared to the left and right wing.

The procedure to consider the aircraft divided into smaller sections is also employed in the literature on wake vortices as the strip model (Hahn and Schwarz 2007). This paper also includes measurements of vertical and horizontal acceleration when an aircraft passes through the counter-rotating wake vortices generated by another aircraft.

As discussed below, there is a hierarchy of models of both aircraft and vortices and in this paper the models presented are at the simple end. The advantage of such an approach is that the results are relatively straightforward to interpret. Future work should include comparisons of relatively simple models such as those described in the current paper with more complex models (of both aircraft and vortices) so that the effect of the extra complexity can be both quantified and understood.

Several vortex models have been employed for wake vortices (Gerz et al. 2002; Ahmad et al. 2014). They typically consist of one (or two) cores which contain vorticity surrounded by an irrotational outer flow region. Such vortex models could also be studied using our methodology. In (Spilman and Stengel 1995), the Lamb-Oseen vortex model was used for an aircraft on final approach to landing.

Multiple co-rotating vortices have been modelled (Parks et al. 1985; Mehta 1987), and counter-rotating vortices have been identified as well (Misaka et al. 2008). 
It would be interesting for us to obtain high-quality aircraft acceleration measurements to make a fit to our model with several co- and/or counterrotating vortices.

A more complete description of the aircraft motion would include e.g. lift and drag forces as presented in (Fischenberg 2010; Schwarz et al. 2010). Depending on the response of the aircraft, it may spend more or less time inside the vortex tube than our model indicates.

\section{Conclusions}

We have extended the modelling of an interaction between an aircraft and a vortex tube to include:

- An arbitrary vortex tube angle

- Both vertical and horizontal acceleration

- An aircraft as having an area (wing and fuselage)

Our model shows that modelling the aircraft as a point will not give a correct acceleration amplitude or duration for cases where the vortex area is of the order of the aircraft area or smaller.

We find it plausible that our model can be used to describe both vortices creating clear-air turbulence (CAT) and wake vortices generated by other aircraft.

\section{Acknowledgements}

We thank a reviewer for providing constructive comments and suggestions, leading to several improvements of the paper.

\section{Conflict of interest}

The author declares that he has no conflict of interest.

\section{References}

Ahmad NN, Proctor FH, Duparcmeur FML, Jacob D (2014) Review of idealized aircraft wake vortex models. AIAA, 52nd ASM, SciTech Forum paper 0927

Airbus (2019) Passenger aircraft. URL https://www.airbus.com/aircraft/passenger-aircraft/a330-family/a330-

Batchelor GK (1967) An Introduction to Fluid Dynamics. Cambridge University Press, UK 
Clark TL, Hall WD, Kerr RM, Middleton D, Radke L, Ralph FM, Neiman PJ, Levinson D (2000) Origins of aircraft-damaging clear-air turbulence during the 9 december 1992 colorado downslope windstorm: Numerical simulations and comparison with observations. Journal of the Atmospheric Sciences 57:1105-1131, DOI 10.1175/1520-0469(2000)057〈1105:OOADCA $\rangle 2.0 . C O ; 2$

Fischenberg A (2010) A method to validate wake vortex encounter models from flight test data. 27th International Congress of the Aeronautical Sciences paper 41

Gerz A, Holzäpfel F, Darracq D (2002) Commercial aircraft wake vortices. Progress in Aerospace Sciences 38:181-208, DOI 10.1016/S0376-0421(02) 00004-0

Hahn KU, Schwarz CW (2007) Safe limits for wake vortex penetration. AIAA Guidance, Navigation and Control Conference and Exhibit paper 6871, DOI $10.2514 / 6.2007-6871$

Jacobson ID, Kuhlthau AR, Richards LG, Conner DW (1978) Passenger ride quality in transport aircraft. Journal of Aircraft 15:724-730, DOI 10.2514/ 3.58438

Kaplan ML, Huffman AW, Lux KM, Cetola JD, Charney JJ, Riordan AJ, Lin YL, Waight III KT (2004) Characterizing the severe turbulence environments associated with commercial aviation accidents. part 2: Hydrostatic mesoscale numerical simulations of supergradient wind flow and streamwise ageostrophic frontogenesis. Meteorology and Atmospheric Physics 88:153173, DOI 10.1007/s00703-004-0079-6

Lamb H (1932) Hydrodynamics. Cambridge University Press, UK

Lin YL (2007) Mesoscale Dynamics. Cambridge University Press, UK

Lunnon R (2016) Turbulence events interpreted by vortex rolls. In: Sharman R, Lane T (eds) Aviation Turbulence, Springer International Publishing, Switzerland, chap 4, DOI 10.1007/978-3-319-23630-8

Mehta RS (1987) Modelling clear-air turbulence with vortices using parameteridentification techniques. Journal of Guidance, Control, and Dynamics 10:27-31, DOI 10.2514/3.20176

Misaka T, Obayashi S, Endo E (2008) Measurement-integrated simulation of clear air turbulence using a four-dimensional variational method. Journal of Aircraft 45:1217-1229, DOI 10.2514/1.34111

Parks EK, Wingrove RC, Bach RE, Mehta RS (1985) Identification of vortexinduced clear air turbulence using airline flight records. Journal of Aircraft 22:124-129, DOI 10.2514/3.45095

Pope SB (2000) Turbulent Flows. Cambridge University Press, UK

Roach WT (1970) On the influence of synoptic development on the production of high level turbulence. Quarterly Journal of the Royal Meteorological Society 96:413-429, DOI 10.1002/qj.49709640906

Saffman PG (1992) Vortex Dynamics. Cambridge University Press, UK

Schwarz CW, Hahn KU, Fischenberg D (2010) Wake encounter severity assessment based on validated aerodynamic interaction models. AIAA Guidance, Navigation and Control Conference paper 7679, DOI 10.2514/6.2010-7679 
Sharman R (2016) Nature of aviation turbulence. In: Sharman R, Lane T (eds) Aviation Turbulence, Springer International Publishing, Switzerland, chap 1, DOI 10.1007/978-3-319-23630-8

Sharman R, Lane T (eds) (2016) Aviation Turbulence, Springer International Publishing, Switzerland, DOI 10.1007/978-3-319-23630-8

Spilman DR, Stengel RF (1995) Jet transport response to a horizontal wind vortex. Journal of Aircraft 32:480-485, DOI 10.2514/3.46745

Wingrove RC, Bach RE (1994) Severe turbulence and maneuvering from airline flight records. Journal of Aircraft 31:753-760, DOI 10.2514/3.46557 